\title{
Mutation of a common amino acid in NKX2.5 results in dilated cardiomyopathy in two large families
}

\author{
Alan Hanley ${ }^{1,3}$, Katie A. Walsh², Caroline Joyce ${ }^{2}$, Michael A. McLellan', Sebastian Clauss', Amaya Hagen, \\ Marisa A. Shea', Nathan R. Tucker ${ }^{1,3}$, Honghuang Lin ${ }^{4}$, Gerard J. Fahy ${ }^{2}$ and Patrick T. Ellinor ${ }^{1,3^{*}}$
}

\begin{abstract}
Background: The genetic basis for dilated cardiomyopathy (DCM) can be difficult to determine, particularly in familial cases with complex phenotypes. Next generation sequencing may be useful in the management of such cases.

Methods: We report two large families with pleiotropic inherited cardiomyopathy. In addition to DCM, the phenotypes included atrial and ventricular septal defects, cardiac arrhythmia and sudden death. Probands underwent whole exome sequencing to identify potentially causative variants.

Results: Each whole exome sequence yielded over 18,000 variants. We identified distinct mutations affecting a common amino acid in NKX2.5. Segregation analysis of the families support the pathogenic role of these variants.

Conclusion: Our study emphasizes the utility of next generation sequencing in identifying causative mutations in complex inherited cardiac disease. We also report a novel pathogenic NKX2.5 mutation.
\end{abstract}

Keywords: Sudden cardiac death, Cardiomyopathy, Arrhythmia, Next generation sequencing

\section{Background}

Dilated cardiomyopathy (DCM) is a condition associated with significant morbidity and mortality that may have identifiable causes or may be idiopathic or inherited. A spectrum of genetic loci associated with DCM has been identified, with over 30 genes implicated [1].

We sought to identify the causative mutation in two unrelated individuals with DCM. Each reported an extensive family history of congenital heart disease (CHD) with multiple cardiac diagnoses affecting several family members. In addition to identifying potential causative mutations, we also sought to address the possibility of a founder effect as both families lived in the same rural geographic area.

To accomplish these goals, we took advantage of the increasing availability and rapidly decreasing cost of next

\footnotetext{
* Correspondence: ellinor@mgh.harvard.edu

${ }^{1}$ Cardiovascular Research Center, Massachusetts General Hospital, Boston, MA, USA

${ }^{3}$ Program in Medical and Populations Genetics, Broad Institute, Cambridge, MA, USA

Full list of author information is available at the end of the article
}

generation sequencing to identify variations within the protein coding region of the genome. We describe the identification of variants potentially implicated in the cardiac disease affecting both families. We also provide a description of our approach to prioritizing variants for further segregation analysis, and the subsequent discovery of a novel NKX2-5 variant.

\section{Case presentations}

A female in her early forties (family 186) presented acutely to hospital with pulmonary edema, atrial fibrillation and non-sustained ventricular tachycardia (VT). She had no prior history of cardiac disease. Echocardiography demonstrated severely impaired left ventricular function. Her son and father had both undergone pacemaker implantation at the age of fifteen and in the fourth decade of life respectively. In addition, there was a history of cardiac disease in the extended family including DCM, atrial and ventricular septal defects and sudden death (Fig 1, Table 1). 


\section{Family 186}

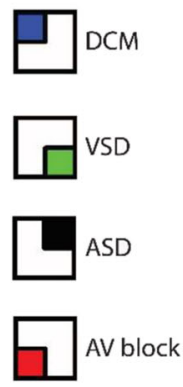

AV block

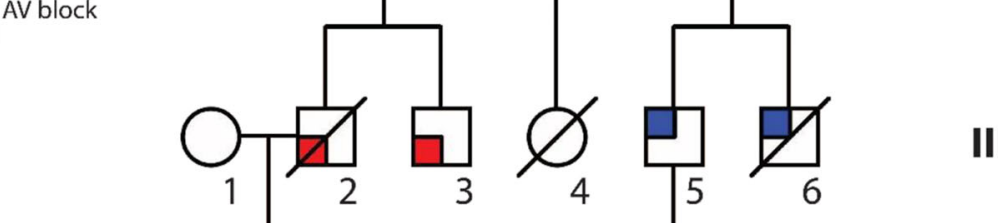

III

IV
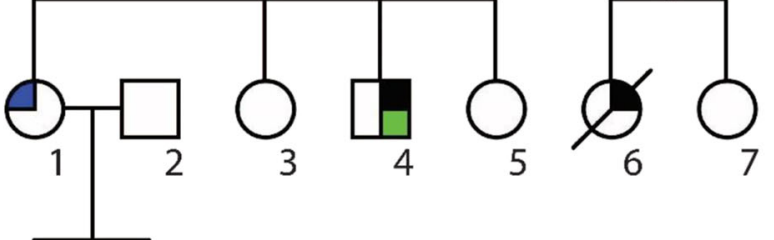

$\square_{1}{ }_{2}$

WT

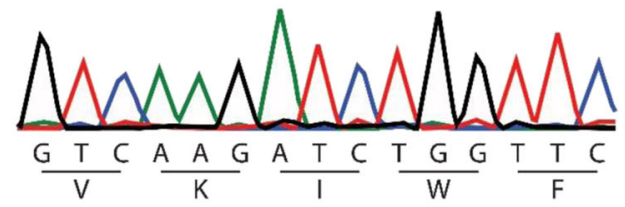

I184F

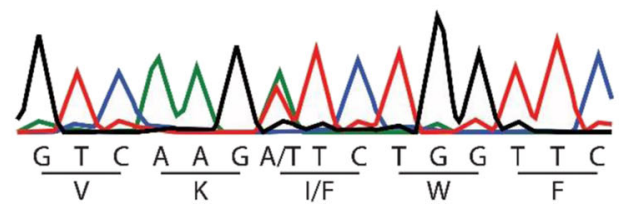

Fig. 1 Pedigree showing affected and unaffected individuals in family 186. Alignment chromatograms showing representative wildtype (WT, above) and heterozygous mutant (I184F, below) tracings

A second unrelated proband (family 187) presented to the same practice in her teenage years with DCM and VT treated with an implantable cardioverterdefibrillator. She also had severely impaired left ventricular function as demonstrated on echocardiography. Family history was remarkable for cardiac disease including DCM, atrial and ventricular septal defects and sudden death (Fig 2, Table 2).

Both families lived in adjacent rural towns with populations of approximately 11,600 and 1,650 people, respectively. Due to the similarities in phenotypes and small referral population we suspected a founder effect may be present, with a common mutation accounting for the observed illnesses. The families were unable to identify a common ancestor, despite well-documented pedigrees extending to the mid-nineteenth century.

\section{Ethics approval and consent to participate}

All participants were enrolled in the ongoing genetics of cardiovascular disease study at Massachusetts General Hospital (MGH). The study was approved by the Institutional Review Board and Human Research Committee at 
Table 1 Clinical characteristics of family 186

\begin{tabular}{lll}
\hline Pedigree & Genotype & Clinical data \\
\hline V1 & + & CHB, PPM, ASD \\
V2 & + & 1 st degree AVB \\
IV.4 & + & AVSD, AF \\
IV.3 & + & Normal \\
IV.5 & + & Normal \\
III.2 & NA & PPM \\
III.3 & NA & PPM \\
II.3 & NA & PPM \\
II.4 & NA 6 & SCD \\
III.5 & NA & SCD \\
IV.6 & NA & DCM, VT \\
III.6 & NA & ASD, SCD \\
\hline
\end{tabular}

MGH and complied with the Declaration of Helsinki. Written informed consent, including consent to publish was obtained prior to performing the evaluations. The probands agreed to genetic testing by way of next generation sequencing in an effort to identify a causative mutation. Samples were processed with the Roche Large Volume DNA isolation kit using magnetic bead technology, following which genomic DNA was extracted on the Roche MagnaPure Automated DNA extractor.

\section{Methods}

Exome capture was performed using Agilent SureSelect assay (v4) according to the manufacturer's recommendation. The library was then amplified and pair-end sequenced by the Illumina HiSeq 2500 platform at the Broad Institute (Cambridge, MA). The BWA software package (Version: 0.5.8) was used to map the sequenced reads to the human reference genome (NCBI Build 37,

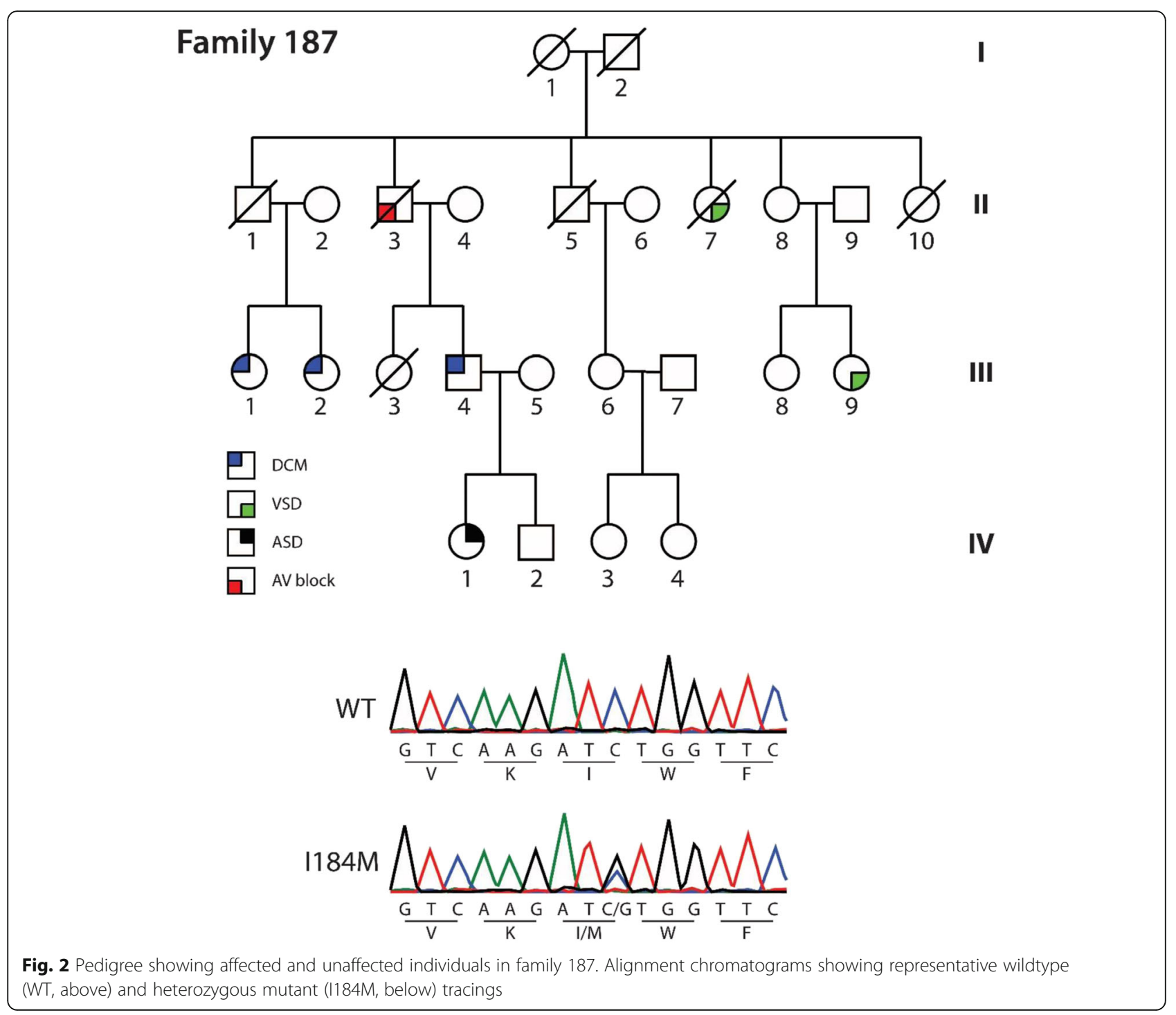


Table 2 Clinical characteristics of family 187

\begin{tabular}{lll}
\hline Pedigree & Genotype & Clinical data \\
\hline$\| I .2$ & + & DCM \\
$\| I .4$ & + & DCM, heart transplant \\
IV.1 & + & AVSD \\
$\| I .6$ & - & VT \\
$\| 1.9$ & - & VSD \\
$\| .1$ & NA & PPM/ICD \\
$\| .3$ & NA & PPM, SCD \\
$\| .5$ & NA & SCD, CAD \\
$\| .7$ & NA & Tricuspid atresia, right ventricular \\
$\| .10$ & hypoplasia, PS, VSD \\
$\| I .3$ & NA & Cyanotic heart disease, SCD \\
\hline
\end{tabular}

hg19) [2]. The resulted SAM files were then converted into BAM files by samtools (Version: 0.1.18) [3]. The MarkDuplicates function of the Picard software package was used to remove duplicate reads, which were defined as those with the exact same start and end positions. The GATK software package (Version 1.2-21) was used to recalibrate base qualities and perform local realignment around indels. The variant calling was implemented by the UnifiedGenotyper function within GATK software package [4].

Sequencing identified in excess of 18,000 variants in each exome. Filtration and prioritization of the variants was performed according to previously published methods [5]. First, all common variants $(>1 \%)$ identified in publically available databases (dbSNP [6], 1000 Genomes Project [7], and Exome Variant Server [8]) were removed from further analyses. Second, synonymous variants were excluded on the basis that these would have no effect on protein function. Following this, analysis of evolutionary conservation of the altered amino acids was performed using the Phylop tool. PolyPhen-2, and SNAP were used to predict the effect of the amino acid substitution on protein structure. Variants affecting amino acids that are highly conserved through evolution and those predicted to have a significant effect on protein structure were then initially analyzed based on their known association with human disease.

\section{Results}

An initial review of the observed variants revealed distinct missense heterozygous mutations in NKX2.5 affecting the same residue in both families. These variants were chosen for further study due to the known role that mutations in NKX2.5 play in inherited cardiovascular disease in humans. None of the other variants had such a clear, previously identified association with the observed phenotypes.

The residue, Isoleucine184, is found in the important homeodomain region and is highly conserved. The variant affecting family 186 was novel, affecting an alignment site with a Phylop conservation score of 4.5 and leading to the amino acid substitution I184F. This variant is predicted by Polyphen-2 to be probably damaging with a score of 1 . Exome variant analysis in family 187 revealed a mutation at a site with a Phylop conservation score of 1.4 , leading to the substitution I184M. The Polyphen score was also 1, with a high probability of being damaging. This variant has been previously described in a family with DCM, left ventricular noncompaction, atrial and ventricular septal defects and conduction system disease [9]. Investigation of the functional effect of this substitution revealed impaired DNA binding with lowered transcriptional activity.

Following identification of these potentially pathogenic mutations in the probands, extended family members underwent clinical evaluation and PCR-based genotyping. Seventeen members of family 186 and nine members of family 187 underwent a standardized interview and physical examination. 12-lead electrocardiogram and echocardiogram were performed where clinically indicated, or in case of a mutation being identified. Affected individuals were identified as those with a self-reported history of cardiac disease such as septal defect closure in infancy, conduction system or rhythm abnormalities on the electrocardiogram or evidence of septal defect or DCM during echocardiographic analysis.

\section{Availability of data and materials}

Disease variants discovered in the present study will be uploaded to the Database of Genotypes and Phenotypes (dbGaP), Bethesda (MD): National Center for Biotechnology Information, National Library of Medicine. Available from: http://www.ncbi.nlm.nih.gov/sites/entrez?db=gap

\section{Confirmation sequencing}

Sanger sequencing was used to confirm the presence of variants and to perform segregation analysis. Oligonucleotide primers flanking the putative variants were designed using genomic sequences from the UCSC Genome Browser (hg19 assembly). Standard conditions were used to perform PCR. DNA sequencing was performed using the ABI PRISM dye terminator method (Applied Biosystems, CA, USA).

\section{Segregation analysis}

In family 186, the proband is individual IV.1. Her sons (V.1 and V.2) demonstrated evidence of cardiac disease. V.1 had a history of conduction system disease requiring pacemaker implant at the age of 15 . At the screening visit a septal defect was apparent on echocardiography, with the atrial pacemaker lead crossing through it to the left atrium. V.2 had first degree heart block evident on 12-lead ECG. In addition, individual IV.4 had a cardiac septal defect repaired surgically in infancy. At the screening visit he was found to be in atrial fibrillation, with mildly impaired LV function on echo. All of these 
affected individuals were found to carry the I184F variant. Other individuals (IV.3, IV.5) harboring the mutation had normal cardiac investigations, despite being older than other clinically affected carriers, indicating incomplete penetrance. Individual IV.4 had two young children (not shown) who are also carriers without evidence of disease, possibly due to their young age.

Several affected individuals were unavailable for genetic testing. Specifically, the proband's father, III. 2 had a pacemaker implanted while in his fourth decade. His brother, III.3 also had a pacemaker. A paternal great-uncle, II.3, had a pacemaker and his daughter, III.4, suffered sudden death at the age of nineteen. Individual II.6 died suddenly at age 3. III.5 was diagnosed with DCM and VT. His daughter, IV.6 had an atrial septal defect and died suddenly at the age of 15 . Her paternal uncle, III.6 had DCM and AF, and died suddenly aged 51 .

In family 187, the proband is individual III.1. Her sister (III.2) was diagnosed with DCM just prior to enrollment. A first-degree cousin, III.4, had a history of DCM requiring transplantation. His daughter (IV.1) had a septal defect corrected surgically in infancy. These affected individuals were all carriers of the I184M variant. Other members of the pedigree had cardiac disease but did not carry the variant. Individual III.6 had a history of nonsustained polymorphic VT documented on 24 hour Holter monitor. This may have been related to extreme caffeine intake at the time of the recording. Her father who died suddenly at a young age (II.5) had documented coronary disease at post mortem examination which was listed as the cause of death. Individual III.9 has a ventricular septal defect, despite not carrying the mutation. As her parents are mutation negative and are both healthy, it is probable that this is a sporadic phenomenon.

Some affected individuals were unavailable for genetic testing; the proband's father (II.1) had a cardiac device implanted. His death was non-cardiac. His brother, II.3 had a pacemaker implanted aged 33 and died suddenly. His sister, II.7 had complex congenital cardiac disease including tricuspid atresia, right ventricular hypoplasia, pulmonary stenosis and a ventricular septal defect requiring a bidirectional Glenn shunt. Individual II.10 had cyanosis and died suddenly in infancy. Another sibling (not shown) was stillborn. Individual III.3 died suddenly aged 22.

\section{Discussion}

NKX2.5 is a highly conserved homeobox transcription factor that has a crucial role in cardiac development and homeostasis, specifying critical elements such as troponin $\mathrm{T}$, connexin $43, \beta$-myosin heavy chain and myosin light chain-2 [10, 11]. The gene is located on chromosome 5q34, spanning 2 exons and encoding a 324 amino acid homeodomain-containing protein [12]. Mutations in this gene cause a phenotypically broad array of structural cardiac defects and arrhythmias with variable penetrance [13]. The most common of these include septal defects and conduction system disease, supported by a recent review of reported mutations [14-16]. While we observed these phenotypes among families 186 and 187, the index cases presented with dilated cardiomyopathy. This was also the most common diagnosis among the extended pedigrees. We also observed incomplete penetrance, the cause for which is poorly understood but may relate to maternal factors [17].

On the basis of the conservation score, expression profile, predicted effect on protein function and segregation with disease in the families, I184F-NKX2.5 and I184MNKX2.5 represent compelling candidate variants for the broad spectrum of cardiac disease in families 186 and 187 respectively. Previously reported functional studies provide further evidence to support the role of variants in I184-NKX2.5 in congenital cardiac disease [9].

The management of families with complex inherited cardiac disease is particularly challenging. Clinical testing can only diagnose mutation carriers expressing a clear phenotype. As mutations in some genes-including NKX2.5-may exert their effect in a time-dependent manner, longitudinal screening of unaffected individuals may be required [18]. Presentation may also include sudden death, and in the absence of a genetic diagnosis it can be difficult to identify at-risk individuals. An informative genetic test may therefore facilitate targeted screening and spare mutation negative individuals from unnecessary follow up and investigation.

In terms of achieving a molecular diagnosis, the pleiotropic nature of the conditions affecting families 186 and 187 does not implicate a particular gene or set of genes. The overall yield of genetic testing for cardiomyopathies employing current panels is $50-60 \%$ in the best cases (such as arrhythmogenic right ventricular cardiomyopathy, or familial hypertrophic cardiomyopathy [19-21]). Often the yield is either significantly lower than $50 \%$ or may be unknown [22]. The yield for testing in channelopathies is similarly unimpressive, with the lone exception of long QT syndrome [22]. In addition, NKX2.5 is not included in most commercially available diagnostic gene panels for either cardiomyopathy or channelopathy [23-25].

Relying on traditional diagnostic methods would therefore have failed to yield a genetic diagnosis in families 186 and 187. Next generation sequencing provides a rapid and unbiased approach of evaluating the exome, greatly expanding the scope of genes in which mutations can be identified. This allowed identification of NKX2.5 variants in each of our probands. Identification of these variants had important diagnostic and management implications for the extended family. The discovery facilitated cascade screening and enabled us to both arrange appropriate 
surveillance for otherwise healthy mutation positive individuals, and conversely to reassure and discharge healthy mutation negative individuals from the clinic.

It is a remarkable coincidence that two families living so close to each other with such a rare inherited disease did not share the same mutation, refuting our initial suspicion that a founder mutation would be discovered. An additional coincidence is the genomic proximity of the variants found in each study family with distinct mutations affecting not only the same rarely-implicated gene but also the same residue.

\section{Conclusion}

Next generation sequencing and in silico variant prioritization may allow for rapid identification of pathogenic and novel mutations in complex pedigrees with pleiotropic cardiac disease. I184F-NKX2.5 is a novel variant associated with congenital heart disease.

\section{Abbreviations}

AF: Atrial fibrillation; CHD: Congenital heart disease; DCM: Dilated cardiomyopathy; DNA: Deoxyribonucleotide acid; ECG: Electrocardiogram; LV: Left ventricle; MGH: Massachusetts General Hospital; PCR: Polymerase chain reaction; UCSC - University of California: Santa Cruz; VT: Ventricular tachycardia

\section{Acknowledgements}

We are deeply grateful to families involved for the time and effort they contributed to the present study.

\section{Funding}

This study was supported by fellowship support from the Irish Cardiac Society (Hanley), an institutional T32 award, T32HL007208 (Tucker), 2R01HL092577, R01HL104156, and K24HL105780 (Ellinor), an American Heart Association Established Investigator Award 13EIA14220013 (Ellinor), and from the Fondation Leducq (14CVD01).

\section{Availability of data and materials}

Disease variants discovered in the present study will be uploaded to the Database of Genotypes and Phenotypes (dbGaP), Bethesda (MD): National Center for Biotechnology Information, National Library of Medicine. Available from: https://www.ncbi.nlm.nih.gov/gap.

\section{Authors' contributions}

AH: Enrolled participants, acquired samples, performed genotyping reactions and sequence alignment, drafted manuscript. KAW: Identified and enrolled participants, acquired samples. CJ: DNA extraction from whole blood. MAM: Sample QC, performed genotyping reactions and sequence alignment. SC, $\mathrm{AH}$ : Assisted with genotyping reactions and sequence alignment. MAS: Curated pedigrees, prepared figures. NRT: Participated in manuscript and figure preparation. HL: NGS data analysis including variant calling. GJF and PTE: Conceived the study and participated in its design and coordination, prepared manuscript. All authors read and approved the final manuscript.

\section{Competing interests}

Dr. Ellinor is the principal investigator on a grant from Bayer HealthCare to the Broad Institute regarding the genetics and therapeutics of atrial fibrillation.

Consent for publication, ethics approval and consent to participate The study was approved by the Institutional Review Board and Human Research Committee at MGH and complied with the Declaration of Helsinki. Written informed consent, including consent to publish was obtained prior to performing the evaluations.

\section{Author details}

Cardiovascular Research Center, Massachusetts General Hospital, Boston, MA USA. ${ }^{2}$ Cork University Hospital, Cork, Wilton, Ireland. ${ }^{3}$ Program in Medical and Populations Genetics, Broad Institute, Cambridge, MA, USA. ${ }^{4}$ Department of Medicine, Boston University School of Medicine, Boston, MA, USA.

Received: 16 June 2016 Accepted: 10 November 2016

Published online: 17 November 2016

\section{References}

1. Hershberger RE, Morales A, Siegfried JD. Clinical and genetic issues in dilated cardiomyopathy: a review for genetics professionals. Genet Med. 2010;12(11):655-67. doi:10.1097/GIM.0b013e3181f2481f.

2. Li H, Durbin R. Fast and accurate long-read alignment with BurrowsWheeler transform. Bioinformatics. 2010;26(5):589-95. doi:10.1093/ bioinformatics/btp698.

3. Li H, Handsaker B, Wysoker A, et al. The sequence alignment/Map format and SAMtools. Bioinformatics. 2009;25(16):2078-9. doi:10.1093/ bioinformatics/btp352.

4. McKenna AH, Hanna M, Banks E, et al. The genome analysis toolkit: a MapReduce framework for analyzing next-generation DNA sequencing data. Genome Res. 2010;20(9):1297-303. doi:10.1101/gr.107524.110.

5. Li M-X, Gui H-S, Kwan JSH, Bao S-Y, Sham PC. A comprehensive framework for prioritizing variants in exome sequencing studies of Mendelian diseases. Nucleic Acids Res. 2012;40(7), e53. doi:10.1093/nar/gkr1257.

6. Home - SNP - NCBI. https://www.ncbi.nlm.nih.gov/snp. Accessed 20 Jan 2016.

7. Abecasis GR, Altshuler D, Auton A, et al. A map of human genome variation from population-scale sequencing. Nature. 2010;467(7319):1061-73. doi:10.1038/nature09534.

8. Exome Variant Server. http://evs.gs.washington.edu/EVS/. Accessed 20 Jan 2016.

9. Costa MW, Guo GL, Wolstein O, et al. Functional characterization of a novel mutation in NKX2-5 associated with congenital heart disease and adultonset cardiomyopathy. Circ Genet. 2013;6(3):238-47. doi:10.1161/ Circgenetics.113.000057.

10. Akazawa H, Komuro I. Cardiac transcription factor Csx/Nkx2-5: Its role in cardiac development and diseases. Pharmacol Ther. 2005;107(2):252-68. doi:10.1016/j.pharmthera.2005.03.005.

11. Tong Y-F. Mutations of NKX2.5 and GATA4 genes in the development of congenital heart disease. Gene. 2016;588(1):86-94. doi:10.1016/j.gene.2016.04.061.

12. International Human Genome Sequencing Consortium. Finishing the euchromatic sequence of the human genome. Nature. 2004;431(7011): 931-45. doi:10.1038/nature03001.

13. Reamon-Buettner SM, Borlak J. NKX2-5: An update on this hypermutable homeodomain protein and its role in human congenital heart disease (CHD). Hum Mutat. 2010;31(11):1185-94. doi:10.1002/humu.21345.

14. Biben C, Weber R, Kesteven S, et al. Cardiac septal and valvular dysmorphogenesis in mice heterozygous for mutations in the homeobox gene Nkx2-5. Circ Res. 2000;87(10):888-95. doi:10.1161/01.RES.87.10.888.

15. Pashmforoush M, Lu J, Chen H, Amand T. Nkx2-5 pathways and congenital heart disease: Loss of ventricular myocyte lineage specification leads to progressive cardiomyopathy and complete heart. Cell. 2004;117:373-86. doi:10.1016/S0092-8674(04)00405-2

16. Ellesøe SG, Johansen MM, Bjerre JV, Hjortdal VE, Brunak S, Larsen LA. Familial atrial septal defect and sudden cardiac death: identification of a novel NKX2-5 mutation and a review of the literature. Congenit Heart Dis. 2016; 11(3):283-90. doi:10.1111/chd.12317.

17. Schulkey CE, Regmi SD, Magnan RA, et al. The maternal-age-associated risk of congenital heart disease is modifiable. Nature. 2015;520(7546):230-3. doi:10.1038/nature14361.

18. Takeda $M$, Briggs LE, Wakimoto $H$, et al. Slow progressive conduction and contraction defects in loss of Nkx2-5 mice after cardiomyocyte terminal differentiation. Lab Invest. 2009;89(9):983-93. doi:10.1038/labinvest.2009.59.

19. Philips B, Cheng A. 2015 update on the diagnosis and management of arrhythmogenic right ventricular cardiomyopathy. Curr Opin Cardiol. 2016; 31(1):46-56. doi:10.1097/HCO.0000000000000240.

20. Kaski JP, Syrris P, Esteban MTT, et al. Prevalence of sarcomere protein gene mutations in preadolescent children with hypertrophic cardiomyopathy. Circ Cardiovasc Genet. 2009;2(5):436-41. doi:10.1161/CIRCGENETICS.108.821314.

21. Andersen PS, Havndrup O, Hougs L, et al. Diagnostic yield, interpretation, and clinical utility of mutation screening of sarcomere encoding genes in 
Danish hypertrophic cardiomyopathy patients and relatives. Hum Mutat. 2009:30(3):363-70. doi:10.1002/humu.20862.

22. Ackerman MJ, Priori SG, Willems S, et al. HRS/EHRA expert consensus statement on the state of genetic testing for the channelopathies and cardiomyopathies: this document was developed as a partnership between the Heart Rhythm Society (HRS) and the European Heart Rhythm Association (EHRA). Europace. 2011;13(8):1077-109. doi:10.1093/europace/eur245.

23. Cardiomyopathy Panel - Genetic Testing Company | The DNA Diagnostic Experts | GeneDx. https://www.genedx.com/test-catalog/available-tests/ cardiomyopathy-panel/. Accessed 20 Jan 2016.

24. Pan Cardiomyopathy Panel | Partners Personalized Medicine. http:// personalizedmedicine.partners.org/Laboratory-For-Molecular-Medicine/ Tests/Cardiomyopathy/PanCardiomyopathy-Panel.aspx. Accessed 20 Jan 2016.

25. Transgenomic - Cardiology Tests. http://www.transgenomic.com/services/ genetic-testing/cardiology-tests/. Accessed 20 Jan 2016.

Submit your next manuscript to BioMed Central and we will help you at every step:

- We accept pre-submission inquiries

- Our selector tool helps you to find the most relevant journal

- We provide round the clock customer support

- Convenient online submission

- Thorough peer review

- Inclusion in PubMed and all major indexing services

- Maximum visibility for your research

Submit your manuscript at www.biomedcentral.com/submit
Biomed Central 